\title{
Soft fluctuating surfactant membranes in supercritical $\mathrm{CO}_{2}$-microemulsions
}

\author{
Olaf Holderer, ${ }^{a}$ Michael Klostermann, ${ }^{* b}$ Michael Monkenbusch, ${ }^{a}$ Ralf Schweins, ${ }^{c}$ \\ Peter Lindner, ${ }^{c}$ Reinhard Strey, ${ }^{b}$ Dieter Richter $^{a}$ and Thomas Sottmann ${ }^{b}$
}

\author{
Received 15th July 2010, Accepted 1st October 2010 \\ DOI: $10.1039 /$ c0cp01199a
}

The bending rigidity of surfactant membranes in novel bicontinuous $\mathrm{CO}_{2}$-microemulsions of the type $\mathrm{H}_{2} \mathrm{O} / \mathrm{NaCl}-\mathrm{scCO}_{2}-\mathrm{Zonyl}$ FSH/Zonyl FSN 100 was determined using both high pressure small angle neutron scattering and neutron-spin echo spectroscopy. As an important result it was found, that the stiffness of the membrane increases solely by an increase of the pressure.

The waste of chemical solvents from chemical processing and related industries represent a huge environmental concern. During the last decades supercritical fluids have attracted much attention as potential replacements for these conventional organic solvents. Supercritical $\mathrm{CO}_{2}\left(T_{\mathrm{c}}=31.1{ }^{\circ} \mathrm{C}\right.$, $\left.p_{\mathrm{c}}=72.8 \mathrm{bar}\right)$ is seen as the most promising candidate because it is cheap, abundant, incombustible, non-toxic, bio- and foodcompatible. Unfortunately $\mathrm{scCO}_{2}$ is generally a very poor solvent, in particular for polar and/or high molecular weight solutes. Thus, in applications where both polar and nonpolar components needed to be dissolved, emulsions and microemulsions were used to overcome this severe limitation. ${ }^{1}$ Furthermore, $\mathrm{scCO}_{2}$ microemulsions are also of great interest from a theoretical point of view because, contrary to classical "state of the art" microemulsions, the properties of $\mathrm{scCO}_{2}$ microemulsions can be strongly influenced just by varying the pressure, i.e. the solvent properties of $\mathrm{CO}_{2}$. So far studies on these novel microemulsion systems concentrate on the phase behavior and the microstructure of $\mathrm{CO}_{2}$-rich microemulsions. ${ }^{1-3}$ Recently, we were able to formulate balanced supercritical $\mathrm{CO}_{2}$-microemulsions containing equal volumes of water and $\mathrm{CO}_{2}$ using technical grade $n$-alkyl-polyglycolether- and perfluoroalkyl-polyglycolether-surfactants. ${ }^{4,5}$ The phase behavior of such a microemulsion system $\mathrm{H}_{2} \mathrm{O} / \mathrm{NaCl}-\mathrm{scCO}_{2}$-Zonyl FSH/Zonyl FSN 100 is shown in Fig. 1 for pressures of $p=160$ (top), 220 (middle) and 300 bar (bottom) as a function of the overall surfactant mass fraction $\gamma$ and the temperature $T^{5}$ Thereby the mass fraction $\alpha$ of $\mathrm{CO}_{2}$ in the mixture of water and $\mathrm{CO}_{2}$ was adjusted to $\alpha=0.40$ and the surfactant mixture consists of equal amounts of Zonyl FSH and Zonyl FSN $100(\delta=0.50) . \mathrm{NaCl}(1 \mathrm{wt} \%$ in the mixture of water and $\mathrm{NaCl} ; \varepsilon=0.01)$ was added to screen possible electrostatic interactions induced by ionic impurities.

\footnotetext{
${ }^{a}$ Institut für Festkörperforschung, Forschungszentrum Jülich, 52425 Jülich, Germany. E-mail: O.Holderer@fz-juelich.de; Fax: + 49 (0)89289 10797; Tel: + 49 (0)89289 10707

${ }^{b}$ Department of Chemistry, University of Cologne, 50939 Cologne, Germany.E-mail: Michael.Klostermann@uni-koeln.de;

Fax: + 49 (0)221 470 5104: Tel: + 49 (0)221470 4548

${ }^{c}$ Institut Laue-Langevin, LSS Group, 6 rue Jules Horowitz, F-38042 Grenoble, France
}

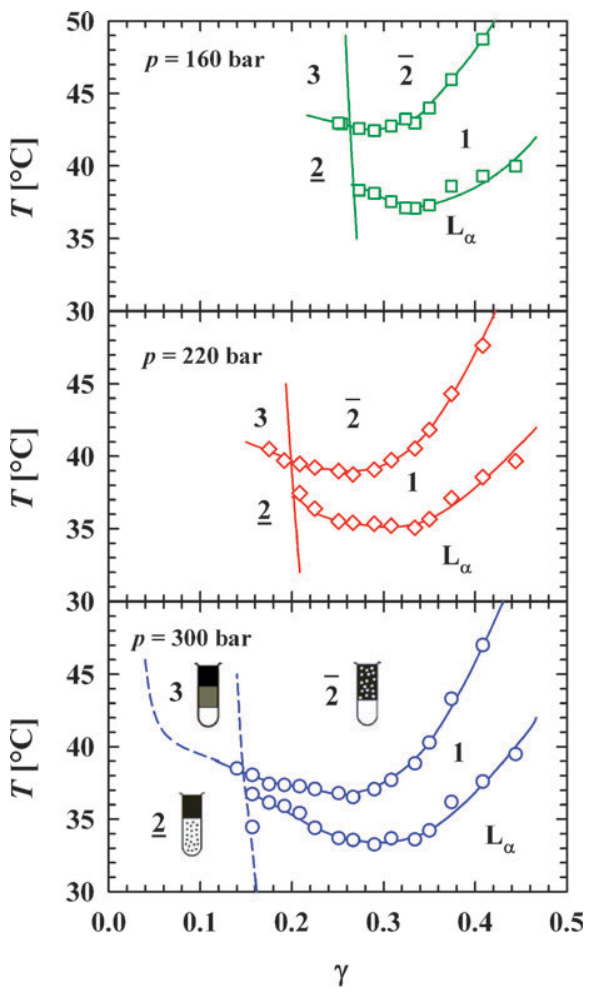

Fig. 1 Phase behaviour of the system $\mathrm{H}_{2} \mathrm{O} / \mathrm{NaCl}(\varepsilon=0.01)$ $\mathrm{scCO}_{2}-$ Zonyl FSH/Zonyl FSN $100(\delta=0.50)$ at a constant $\mathrm{CO}_{2}$ to water plus $\mathrm{CO}_{2}$ ratio of $\alpha=0.40$. To study the influence of the pressure on the phase boundaries $T(\gamma)$-sections through the phase prism are performed at three different pressures, i.e. at $p=160,220$ and 300 bar.

As can be seen in Fig. 1 the phase behavior of this system resemble the phase behavior of well-known non-ionic microemulsions at ambient pressure. ${ }^{6}$ Thus, at low temperatures a $\mathrm{CO}_{2}$-in-water microemulsion coexists with a $\mathrm{CO}_{2}$-excess phase (2), while at high temperatures a coexistence of a water-in- $\mathrm{CO}_{2}$ microemulsion with a water-excess phase $(\overline{2})$ is found. This phase inversion manifests itself in an extended three phase region which can be observed at intermediate temperatures and low surfactant weight fractions $\gamma$. Increasing $\gamma$ the three phase region meets the one phase region at the so-called optimum point $(\tilde{\gamma}, \tilde{T})$. It defines the minimum amount of surfactant needed to solubilize water and $\mathrm{CO}_{2}$ at the phase inversion temperature. The high impact of the pressure onto the phase behavior of $\mathrm{CO}_{2}$-microemulsions is shown in Fig. 1. Increasing the pressure from $p=160$ to 300 bar the optimum point shifts to lower temperatures and significantly lower 


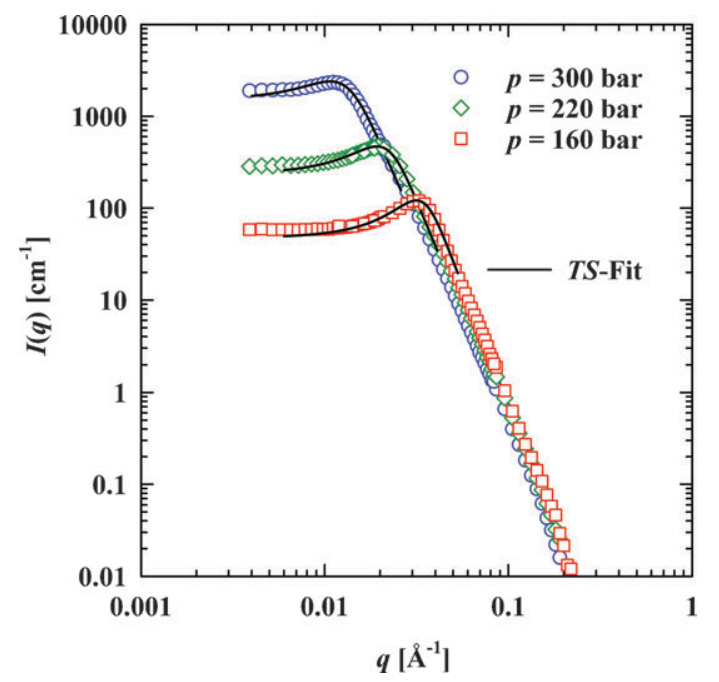

Fig. 2 Scattering curves of the system $\mathrm{D}_{2} \mathrm{O} / \mathrm{NaCl}(\varepsilon=0.01)$ $\mathrm{scCO}_{2}-$ Zonyl FSH/Zonyl FSN $100(\delta=0.50)$ at $\alpha=0.37$ and three different pressures. All curves were measured close to the respective optimum point, i.e. at $p=300$ bar and $(\gamma, T)=\left(0.18,35.0{ }^{\circ} \mathrm{C}\right)$, $p=220$ bar and $(\gamma, T)=\left(0.23,34.8{ }^{\circ} \mathrm{C}\right), p=300$ bar and $(\gamma, T)=\left(0.29,36.4{ }^{\circ} \mathrm{C}\right)$. The solid line represents the Teubner-Strey-fit. ${ }^{12}$ Note that the phase boundaries are shifted to lower temperatures $\left(\Delta T \approx 2 \mathrm{~K}\right.$ ) if $\mathrm{H}_{2} \mathrm{O}$ is replaced with $\mathrm{D}_{2} \mathrm{O}$.

surfactant mass fractions. Thus, the solubilization of water and $\mathrm{CO}_{2}$ by the surfactant becomes increasingly efficient at high pressures. Note that within the one phase region an extended lamellar $\mathrm{L}_{\alpha}$-phase can be found at low temperatures.

The microstructure of these $\mathrm{CO}_{2}$ microemulsions was studied performing small angle neutron scattering (SANS) experiments at the instrument D11 at the Institut Laue-Langevin (Grenoble, France) using a self-constructed high pressure SANS-cell. ${ }^{7}$ These experiments were carried out in almost perfect bulk contrast using $\mathrm{D}_{2} \mathrm{O}$ instead of $\mathrm{H}_{2} \mathrm{O}$ close to the respective optimum points, i.e. at $\gamma=0.18$ ( $p=300$ bar), $\gamma=0.23(p=220$ bar $)$ and $\gamma=0.29(p=160$ bar $)$. The scattering data obtained are shown in Fig. 2. At all three pressures a pronounced correlation peak is observed at intermediate $q$-values followed by a steep $\exp \left(-q^{2} t^{2}\right) q^{-4}$ decrease e,9 $^{8,9}$ of the scattering intensity at high $q$-values. This shape of the scattering curves together with the observed phase inversion strongly supports the existence of a bicontinuous structured $\mathrm{scCO}_{2}$-microemulsion. ${ }^{10,11}$ Analyzing the scattering peak by the model of Teubner and Strey ${ }^{12}$ a periodicity of the water and oil domains of $d_{\mathrm{TS}}=186,301,512 \AA$ as well as a correlation length $\xi_{\mathrm{TS}}=85,109,157 \AA$ is obtained with increasing pressure, i.e. decreasing surfactant mass fraction. Additionally, a rather high monomeric solubility of the fluorosurfactant in $\mathrm{CO}_{2}$ of around $10 \mathrm{wt} \%$ could be extracted from the high $q$-part of the scattering curve using the Porod-model for diffuse interfaces. ${ }^{9}$

In order to understand the strong influence of the pressure on the phase behavior and the microstructure of these novel bicontinuous $\mathrm{CO}_{2}$-microemulsions and motivated by theoretical studies of Safran et al., ${ }^{13}$ Morse, ${ }^{14}$ Golubovic, ${ }^{15}$ and Gompper and $\mathrm{Kroll}^{16}$ we studied the properties of the amphiphilic surfactant film using both SANS and neutron spin echo (NSE) spectroscopy. Following Pieruschka and $\operatorname{Safran}^{13}$ the renormalized bending rigidity $\kappa_{\text {SANS }}$ of the surfactant membrane can be calculated from the structural parameters of the microemulsion using the model of random interfaces by

$$
\frac{\kappa_{\mathrm{SANS}}}{k_{\mathrm{B}} T}=\frac{10 \sqrt{3} \pi}{64} \frac{\xi_{\mathrm{TS}}}{d_{\mathrm{TS}}}
$$

In doing so we found that $\kappa_{\text {SANS }}$ slightly decreases with increasing pressure (and concomitantly decreasing $\gamma$ ) from $\kappa_{\mathrm{SANS}}(160$ bar $)=0.38 k_{\mathrm{B}} T$ to $\kappa_{\mathrm{SANS}}(220$ bar $)=0.31 k_{\mathrm{B}} T$ and $\kappa_{\text {SANS }}(300$ bar $)=0.26 k_{\mathrm{B}} T$. The absolute values of the renormalized bending rigidity $\kappa_{\mathrm{SANS}}$ in these novel bicontinuous $\mathrm{CO}_{2}$-microemulsions correspond almost quantitatively to the values obtained for surfactant membranes in "classical state of the art" microemulsions containing water, $n$-alkane and nonionic surfactant. ${ }^{17}$ However, the renormalized bending rigidity $\kappa_{\text {SANS }}$ lumps together all effects from membrane fluctuations up to a wavelength corresponding to the structural dimension. It differs from the "bare" bending rigidity $\kappa_{\text {bare }}$ of the membrane by a renormalization factor, which depends for a given system on the characteristic length scale $d_{\mathrm{TS}} / 2$ of the microemulsion ${ }^{14-16}$ according to

$$
\frac{\kappa_{\text {bare }}}{k_{\mathrm{B}} T}=\frac{\kappa_{\mathrm{SANS}}}{k_{\mathrm{B}} T}+\frac{3}{4 \pi} \ln \left(\frac{d_{\mathrm{TS}}}{2 l_{\mathrm{c}}}\right)
$$

where $l_{\mathrm{c}}$ is the effective thickness of the amphiphilic film. Based on systematic SANS-studies on $\mathrm{CO}_{2}$-swollen microemulsion droplets we estimated $l_{\mathrm{c}}=15 \pm 2.5 \AA$. Using eqn (2) we found that $\kappa_{\text {bare }}(160$ bar $)=0.82 k_{\mathrm{B}} T, \kappa_{\text {bare }}(220$ bar $)=0.85 k_{\mathrm{B}} T$ and $\kappa_{\text {bare }}(300$ bar $)=0.94 k_{\mathrm{B}} T$.

This trend clearly shows that in bicontinuous $\mathrm{CO}_{2}$-microemulsions the "bare" bending rigidity $\kappa_{\text {bare }}$, i.e. the "stiffness" of a surfactant membrane, increases with increasing pressure. One possible explanation for this trend might be the fact that the density of $\mathrm{CO}_{2}$ increases with increasing pressure leading to a stronger interaction between the solvent $\mathrm{CO}_{2}$ and the fluorinated surfactant tails. The increasing penetration of the $\mathrm{CO}_{2}$-molecules into the surfactant film also explains the shift of the phase boundaries to lower temperatures due to an increasing curvature of the surfactant membrane around the water.

While the "bare" bending rigidity $\kappa_{\text {bare }}$ can only be extracted indirectly from the analysis of the SANS-curves, a direct determination of the elastic properties of surfactant membranes is possible studying the dynamic properties of bicontinuous $\mathrm{CO}_{2}$-microemulsions with the help of quasielastic scattering. Among these techniques neutron spin-echo (NSE) spectroscopy is the one with the highest energy resolution and thus provides a means to study the dynamics of mesoscopic objects. Furthermore the decay time $\tau$ for thermally activated fluctuations of surfactant membrane patches is in the regime of nanoseconds and therewith inside the time window of NSE spectroscopy. The neutron spin-echo experiments on $\mathrm{scCO}_{2}$-microemulsions have been performed at the J-NSE instrument $^{18,19}$ at the FRM II research reactor of the TU München using an inhouse built high-pressure NSE-cell. It delivers the intermediate scattering function $S(q, \tau)$, the Fourier transform of the scattering function $S(q, \omega)$. 
The fluctuation of membrane patches in "classical" microemulsions at ambient pressure has been described by the theory of Zilman and Granek. ${ }^{20}$ It relates the relaxation rate of the membrane $\Gamma_{\mathrm{ZG}}$ to the bending rigidity $\kappa$. Starting from the free bending energy of a surfactant membrane for small deformations ${ }^{20} H=1 / 2 \kappa \int \mathrm{d}^{2} r\left[\nabla^{2} h(\boldsymbol{r})\right]^{2}$, with the deviation $h(\boldsymbol{r})$ from a flat membrane patch, the intermediate scattering function is derived. In an approximation, the intermediate scattering function is described by a stretched exponential function

$$
S(q, \tau) / S(q, 0)=\exp \left(-\left(\Gamma_{\mathrm{ZG}} \cdot \tau\right)^{\beta}\right)
$$

with the relaxation rate

$$
\Gamma_{\mathrm{ZG}}=0.025 \cdot \gamma_{\mathrm{ZG}}\left(\frac{\kappa}{k_{\mathrm{B}} T}\right)^{-0.5} \frac{k_{\mathrm{B}} T}{\eta} q^{3}
$$

where $\eta$ is the viscosity of the solvent that surrounds the surfactant membrane (in this case the average viscosity of water and $\mathrm{CO}_{2}$ is used). In eqn (4) $\gamma_{\mathrm{ZG}}=1-3\left(k_{\mathrm{B}} T / 4 \pi \kappa\right)$ $\ln \left(q \xi_{\mathrm{TS}}\right)$. According to the Zilman-Granek model the stretching exponent $\beta$ is predicted to be $\beta=2 / 3$.

Fig. 3 shows the intermediate scattering function $S(q, \tau)$ of the $\mathrm{D}_{2} \mathrm{O} / \mathrm{NaCl}(\varepsilon=0.01)-\mathrm{scCO}_{2}-$ Zonyl FSH/Zonyl FSN 100 $(\alpha=0.37, \delta=0.50)$ microemulsion near the optimum point at $T=35{ }^{\circ} \mathrm{C}, \gamma=0.23$ and a pressure of $p=220$ bar for four different $q$-values between $q=0.05$ and $q=0.15 \AA^{-1}$ (corresponding to length scales in real space of $d=2 \pi / q=$ $125-42 \AA)$.

Fitting the experimental data with the expression of eqn (3) shows that the $q^{3}$-dependence of the relaxation rate, which is predicted for bicontinuous microemulsions according to eqn (4), is found also for bicontinuously structured supercritical $\mathrm{CO}_{2}$-microemulsion, as well as the typical stretching exponent $\beta=2 / 3$. Compared to "classical" microemulsions consisting of decane, water and $\mathrm{C}_{10} \mathrm{E}_{4}$ (n-decyltetraoxyethylene), which

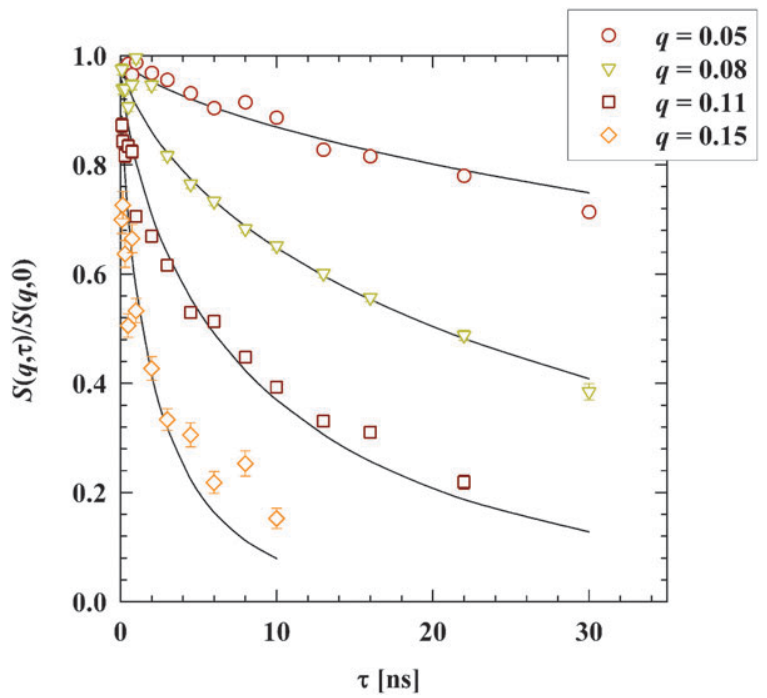

Fig. 3 Intermediate scattering function $S(q, \tau)$ of a $\mathrm{D}_{2} \mathrm{O} / \mathrm{NaCl}$ $(\varepsilon=0.01)-\mathrm{scCO}_{2}-$ Zonyl FSH/Zonyl FSN $100(\delta=0.50)$ microemulsion at $\alpha=0.37, T=35{ }^{\circ} \mathrm{C}, \gamma=0.23$ and a pressure of $p=220$ bar reflecting the membrane dynamics on local length scales. The experimental data are fitted with the numerical solution of eqn (3). ${ }^{21,22}$ form bicontinuous structures at similar surfactant mass fractions, the relaxation rate increases by roughly a factor of two. ${ }^{21}$ The average viscosity, which has about half the value in the supercritical microemulsion compared to the "classical" oil-water microemulsion due to the very low viscosity of $\mathrm{CO}_{2}$ in its supercritical state, partly accounts for this effect.

The values for the bending rigidity $\kappa_{\mathrm{NSE}}$ obtained from the relaxation rate, however, show a strong $q$-dependency at low $q$-values. $\kappa_{\mathrm{NSE}}$ increases from $\kappa_{\mathrm{NSE}}=0.45 k_{\mathrm{B}} T$ at $q=0.05 \AA^{-1}$ to a plateau value of $\kappa_{\mathrm{NSE}}=0.88 k_{\mathrm{B}} T$ at $q=0.15 \AA^{-1}$. A possible explanation for this trend might be the fact that at low $q$-values, where the membrane is studied on a scale which is only somewhat smaller than the characteristic length scale of the microstructure $d_{\mathrm{TS}} / 2$, the relaxation rate is partly influenced by the diffusion of water or $\mathrm{CO}_{2}$ domains. In order to account for this effect the NSE-data were reevaluated under the assumption of a weighted linear combination of the two independent relaxation modes, namely the diffusion of the microemulsion domains and the fluctuations of membrane patches. ${ }^{22}$ Here, the diffusion constant of the domains was roughly estimated from the domain size $d_{\mathrm{TS}} / 2$ applying the Stokes-Einstein relation. Using this assumption the reevaluation of the NSE-data yielded a $q$-independent bending rigidity of around $\kappa_{\mathrm{NSE}} \approx 0.90 k_{\mathrm{B}} T$. The amplitude of the diffusion process is found to decrease rapidly from $A=0.4$ at $q=0.05 \AA^{-1}$ to $A=0.00$ at $q=0.15 \AA^{-1}$.

A quantitative determination of the bending rigidity with the Zilman-Granek theory, however, implies that not all the approximations leading to eqn (3) are carried out, but numerical integrations of the expression of a fluctuating membrane patch are used instead. ${ }^{21,23}$ The intermediate scattering function $S(q, \tau)$ expressed in this framework is presented in ref. 21 and contains the bending rigidity as only fitting parameter. By integrating explicitly over all undulation wave vectors of the membrane one gets $\kappa$ without effects of renormalization. ${ }^{21,23}$

Fig. 4 shows the bending rigidity $\kappa_{\mathrm{NSE}}$ obtained from the NSE experiment using the integral version of the Zilman-Granek model of fluctuating patches as a function of scattering vector $q$. As can be seen the value of the bending rigidity $\kappa_{\mathrm{NSE}}$ slightly increases with $q$. Again, this increase might be attributed to the fact that hydrodynamic contributions from fluctuations of water or $\mathrm{CO}_{2}$ domains decrease with increasing values of $q$. Averaging over all values the bending rigidity is determined to be $\kappa_{\mathrm{NSE}}=0.41 \pm 0.06 k_{\mathrm{B}} T$, which is somewhat smaller than the "bare" bending modulus $\kappa_{\text {bare }}=0.75 k_{\mathrm{B}} T$ obtained from the analysis of SANS experiments on the same sample. Such differences in the absolute value of the bending rigidities determined by SANS and NSE are known from studies of "classical" hydrocarbon microemulsions. ${ }^{21,23}$ In the case of supercritical $\mathrm{CO}_{2}$-microemulsions differences might be led back to a simplified consideration of the viscosity in the Zilman-Granek-model, which uses an average viscosity for the solvent surrounding both sides of the membrane. In classical microemulsions where the viscosities of the polar and the non-polar phase are roughly the same, this approach is valid. In case of supercritical $\mathrm{CO}_{2}$, however, the viscosity of water is almost a factor of 10 higher than that of $\mathrm{scCO}_{2}$ which leads to a highly "asymmetric" viscosity on both sides of the membrane. This fact might give rise to asymmetric 


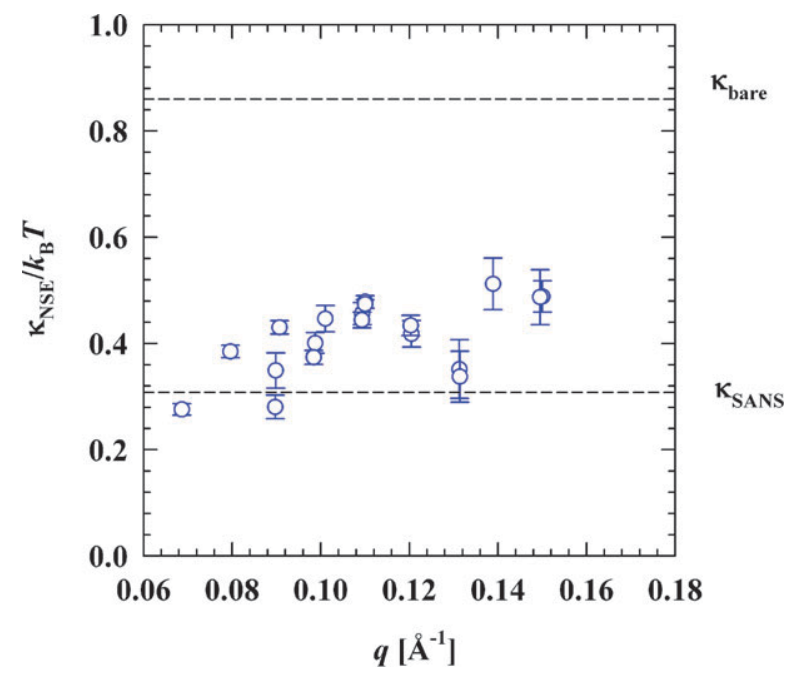

Fig. 4 Bending rigidity $\kappa_{\text {NSE }}$ as a function of $q$ for the $\mathrm{D}_{2} \mathrm{O} / \mathrm{NaCl}$ $(\varepsilon=0.01)-\mathrm{scCO}_{2}-$ Zonyl FSH/Zonyl FSN $100(\delta=0.50)$ microemulsion at $\alpha=0.40, T=35{ }^{\circ} \mathrm{C}, \gamma=0.23$ and a pressure of $p=220$ bar. The dashed lines correspond to the renormalized bending rigidity $\kappa_{\text {SANS }}$ and the "bare" bending rigidity $\kappa_{\text {bare }}$ determined from $d_{\mathrm{TS}}$ and $\xi_{\mathrm{TS}}$ from SANS according to the Gaussian random field approximation. ${ }^{13,26}$

undulations of the amphiphilic film, which are not accounted in the Zilman-Granek-model, leading to the observed differences between $\kappa_{\text {NSE }}$ and $\kappa_{\text {bare }}$. Furthermore, by using the average solvent viscosity the energy dissipation in the surfactant layer due to its viscosity is not taken into account. In previous studies this effect was simply incorporated by the assumption of an effective sample viscosity which is up to 4 times higher than the viscosity of the surrounding solvent and therefore far from reality. ${ }^{24,25}$

In summary we determined the bending rigidity of the amphiphilic film in a bicontinuously structured $\mathrm{scCO}_{2}-$ microemulsion using both high pressure SANS- and high pressure NSE-experiments. The analysis of the SANS measurements according to the Gaussian random field approximation yield the renormalized rigidity $\kappa_{\mathrm{SANS}}$ as well as the renormalization corrected bare bending rigidity $\kappa_{\text {bare }}$ which both correspond almost quantitatively to the values obtained for surfactant membranes in "classical state of the art" microemulsions containing water, $n$-alkane and non-ionic surfactant. Strikingly it is found that the "stiffness" of surfactant membranes in bicontinuous $\mathrm{CO}_{2}$-microemulsions increases with increasing pressure. Complementary high pressure NSE measurements of these novel $\mathrm{scCO}_{2}$-microemulsions provide relaxation rates which are by roughly a factor of two larger than the ones observed in "classical state of the art" microemulsions. The average viscosity, which is a factor of two lower in the $\mathrm{CO}_{2}$ microemulsion partly accounts for this effect. The bending rigidity $\kappa_{\text {NSE }}$ has been determined by applying the ZilmanGranek theory with numerical integration over the undulation mode spectrum. This analysis yields a value of $0.41 k_{\mathrm{B}} T$, which is smaller than the "bare" bending rigidity $\kappa_{\text {bare }}$ obtained from the analysis of the SANS-measurements. Such differences in the absolute value of $\kappa_{\mathrm{NSE}}$ and $\kappa_{\text {bare }}$ might be attributed to a partly simplified consideration of the viscosity in the
Zilman-Granek model, which is based on a similar viscosity on both sides of the membrane. This situation, however, holds not true in $\mathrm{scCO}_{2}$-microemulsion due to the very low viscosity of $\mathrm{scCO}_{2}$.

\section{Acknowledgements}

The authors gratefully thank Lorenz Kramer for his help in the early stages of this study and the SoftComp-Network of excellence for financial support. Michael Klostermann also acknowledges financial support by the International Helmholtz Research School of Biophysics and Soft Matter (IHRS BioSoft).

\section{Notes and references}

1 J. Eastoe, S. Gold and D. C. Steytler, Langmuir, 2006, 22 9832-9842.

2 K. Harrison, J. Goveas, K. P. Johnston and E. A. Orear, Langmuir, 1994, 10, 3536-3541.

3 J. Eastoe, S. Gold, S. Rogers, P. Wyatt, D. C. Steytler, A. Gurgel, R. K. Heenan, X. Fan, E. J. Beckman and R. M. Enick, Angew. Chem., Int. Ed., 2006, 45, 3675-3677.

4 M. Schwan, L. G. A. Kramer, T. Sottmann and R. Strey, Phys. Chem. Chem. Phys., 2010, 12, 6247-6252.

5 M. Klostermann, L. Kramer, T. Sottmann and R. Strey, Langmuir, in preparation.

6 M. Kahlweit and R. Strey, Angew. Chem., Int. Ed. Engl., 1985, 24, 654-668.

7 L. Kramer, PhD thesis, University of Cologne, 2008.

8 G. Porod, in Small Angle X-Ray Scattering, ed. O. Glatter and O. Kratky, Academic Press, New York, 1982.

9 R. Strey, J. Winkler and L. Magid, J. Phys. Chem., 1991, 95, $7502-7507$.

10 S. H. Chen, S. L. Chang and R. Strey, Prog. Colloid Polym. Sci., $1990,81,30-35$.

11 T. Sottmann, R. Strey and S. H. Chen, J. Chem. Phys., 1997, 106, 6483-6491.

12 M. Teubner and R. Strey, J. Chem. Phys., 1987, 87, 3195-3200.

13 P. Pieruschka, S. A. Safran and S. T. Marcelja, Phys. Rev. E: Stat. Phys., Plasmas, Fluids, Relat. Interdiscip., 1995, 52, 1245-1247.

14 D. C. Morse, Phys. Rev. E: Stat. Phys., Plasmas, Fluids, Relat. Interdiscip., 1994, 50, R2423-R2426.

15 L. Golubovic, Phys. Rev. E: Stat. Phys., Plasmas, Fluids, Relat. Interdiscip., 1994, 50, R2419-R2422.

16 G. Gompper and D. M. Kroll, Phys. Rev. Lett., 1998, 81, 2284-2287.

17 T. Sottmann and R. Strey, in Fundamentals of Interface and Colloid Science, ed. J. Lyklema, Elsevier Academic Press, Heidelberg, 2005.

18 M. Monkenbusch, R. Schatzler and D. Richter, Nucl. Instrum. Methods Phys. Res., Sect. A, 1997, 399, 301-323.

19 O. Holderer, M. Monkenbusch, G. Borchert, C. Breunig and K. Zeitelhack, Nucl. Instrum. Methods Phys. Res., Sect. A, 2008, 586, 90-94.

20 A. G. Zilman and R. Granek, Phys. Rev. Lett., 1996, 77, 4788-4791.

21 M. Mihailescu, M. Monkenbusch, H. Endo, J. Allgaier, G. Gompper, J. Stellbrink, D. Richter, B. Jakobs, T. Sottmann and B. Farago, J. Chem. Phys., 2001, 115, 9563-9577.

22 L. R. Arriaga, I. Lopez-Montero, F. Monroy, G. Orts-Gil, B. Farago and T. Hellweg, Biophys. J., 2009, 96, 3629-3637.

23 O. Holderer, H. Frielinghaus, D. Byelov, M. Monkenbusch, J. Allgaier and D. Richter, J. Chem. Phys., 2005, 122, 094908.

24 B. Farago, M. Monkenbusch, K. D. Goecking, D. Richter and J. S. Huang, Physica B., 1995, 213, 712-717.

25 S. Komura, T. Takeda, Y. Kawabata, S. K. Ghosh, H. Seto and M. Nagao, Phys. Rev. E: Stat. Phys., Plasmas, Fluids, Relat. Interdiscip., 2001, 6304, 041402.

26 G. Gompper, H. Endo, M. Mihailescu, J. Allgaier, M. Monkenbusch, D. Richter, B. Jakobs, T. Sottmann and R. Strey, Europhys. Lett., 2001, 56, 683-689. 